\title{
AN INTEGRAL FORMULA FOR MULTIDIMENSIONAL EVOLUTION EQUATIONS
}

\author{
E. C. SVENDSEN
}

\begin{abstract}
This paper contains an irtegral formula for the one-parameter semigroup generated by a sum of contractions of operator-valued tensors. The formula expresses the semigroup in terms of the semigroups generated by the quadratic forms associated with the tensors. It is used to construct solutions of some wave and diffusion equations from solutions of related lower-dimensional equations.
\end{abstract}

1. Introduction. Linear differential operators on $R^{n}$ can be regarded as sums of contractions of operator-valued tensors. This paper contains a formula for the one-parameter semigroup generated by such a sum. The formula expresses the semigroup in terms of the semigroups generated by the quadratic forms associated with the tensors. It is a combination of an integral formula of Kurtz [3] and a product formula of Trotter [5].

The formula is discussed in $\S 2$, and is used in $\S 3$ to construct solutions of some wave and diffusion equations from solutions of related lower-dimensional equations.

2. The main result. Our results are in terms of contraction semigroups on a Banach space $B$. We use these sets of linear operators on $B: \operatorname{Ctr}(B)$, the contractions; $\operatorname{Opr}(D)$, the operators with domain $D$; and $\operatorname{Gen}(D)$, the operators $A$ in $\operatorname{Opr}(D)$ whose closures generate strongly continuous one-parameter semigroups $\exp (A t)$ in $\operatorname{Ctr}(B)$. If $A$ is in $\operatorname{Gen}(D)$ and $F$ is a function from $[0, \infty)$ to $\operatorname{Ctr}(B)$, we say that $\exp (A t) \approx F(t)$ if

$$
\exp (A t) f=\lim _{k \rightarrow \infty} F(t / k)^{k} f
$$

for all $f$ in $B$.

For $j=0,1, \ldots, k$, let $T_{j}$ be a tensor on $R^{n}$ of type $(j, j)$ with values in $\operatorname{Opr}(D)$. As usual, identify $R^{n}$ with its dual. Then $T_{j}$ is a $2 j$-linear form on $R^{n}$ with values in $\operatorname{Opr}(R)$. Let $T_{j}^{2}$ be the multiple quadratic form defined by $T_{j}^{2}(v)=T_{j}(v, v)$ and $\operatorname{Ctr}_{j}\left(T_{j}\right)$ be the multiple contraction defined by

$$
\operatorname{Ctr}_{j}\left(T_{j}\right)=\sum T_{j}^{2}\left(u_{i_{1}}, \ldots, u_{i_{j}}\right),
$$

where $u_{1}, \ldots, u_{n}$ is any orthonormal basis of $R^{n}$ and the sum is over $\{1, \ldots, n\}^{j}$. Finally, let

$$
\operatorname{Ctr}(T)=\sum_{j=0}^{k} \operatorname{Ctr}_{j}\left(T_{j}\right) .
$$

Received by the editors June 9, 1983 and, in revised form, October 31, 1983.

1980 Mathematics Subject Classification. Primary 47D05; Secondary 35L45.

(C) 1984 American Mathematical Society $0002-9939 / 84 \$ 1.00+\$ .25$ per page 
Here is our main result:

THEOREM 1. Suppose that $\operatorname{Ctr}(T)$ is in $\operatorname{Gen}(D)$. Let $\mu_{1}$ be the (unique) probability measure on $S^{n-1}$ that is invariant under orthogonal transformations, and let $\mu_{j}$ be the $j$-fold product of $\mu_{1}$.

(1) For each $j$, let $F_{j}$ be a function from $\left(S^{n-1}\right)^{j} \times[0, \infty)$ to $\operatorname{Ctr}(B)$ with these properties: $F_{j}(u, 0)=I$ for all $u$;

$$
\left(F_{j}(u, t)-\left(I+T_{j}^{2}(u) t\right)\right) f=o(t)
$$

uniformly in $\left(S^{n-1}\right)^{j}$ for all $f$ in $D$ (that and other limits are as $t \rightarrow 0$ ); and $F(, t) f$ is Bochner-integrable for all $t$ and $f$. Then

$$
\exp (\operatorname{Ctr}(T) t) \approx \prod_{j=0}^{k} \int_{\left(S^{n-1}\right)^{j}} F_{j}\left(u, n^{j} t\right) d \mu_{j}(u) .
$$

(2) Suppose that for each $u, T_{j}^{2}(u)$ maps $D$ to itself and is in $\operatorname{Gen}(D)$. Then the function $F_{j}$ defined by

$$
F_{j}(u, t)=\exp \left(T_{j}^{2}(u) t\right)
$$

satisfies the hypotheses of part (1).

(3) If each $T_{j}$ is as in part (2), then

$$
\exp (\operatorname{Ctr}(T) t) \approx \prod_{j=0}^{k} \int_{\left(S^{n-1}\right)^{j}} \exp \left(n^{j} T_{j}^{2}(u) t\right) d \mu_{j}(u)
$$

The reason for part (1) is that some of the $F_{j}$ may not be as in equation (2.2).

Since $T_{j}^{2}(u)$ is invariant under changes in sign of the $u_{i}$, the integrals in (2.3) contain redundancy (as might those in (2.1)). The redundancy is eliminated in this version of Theorem 1:

COROLlaRY 1. Theorem 1 is true if $S^{n-1}$ is replaced by the space $P^{n-1}$ of lines in $R^{n}$ that pass through the origin; $u$ is replaced by $L ; T_{j}^{2}$ is replaced by the function $\tilde{T}_{j}^{2}$ from $\left(P^{n-1}\right)^{j}$ to $\operatorname{Opr}(D)$ defined by $\tilde{T}_{j}^{2}(L)=T_{j}^{2}(u)$, where $u_{i}$ is either unit vector in $L_{i}$; and $\mu_{j}$ is replaced by $\tilde{\mu}_{j}$.

Let $A$ be in $\operatorname{Opr}(D)$ and $F$ be a function from $[0, \infty)$ to $\operatorname{Ctr}(B)$. We say that $\exp (A t) \approx_{1} F(t)$ if $F(0)=I$ and

$$
(F(t)-(I+A t)) f=o(t)
$$

for all $f$ in $D$. (If $A$ is not in $\operatorname{Gen}(D)$, " $\exp (A t)$ " is meaningless by itself.) Our proof of Theorem 1 is based upon this lemma of Chernoff [1]:

Lemma 1. Suppose that $A$ is in $\operatorname{Gen}(D)$. If $\exp (A t) \approx_{1} F(t)$, then $\exp (A t) \approx$ $F(t)$.

We use this version of the Trotter product formula: 
LEMMA 2. For $i=1, \ldots, n$, let $A_{i}$ be in $\operatorname{Opr}(D)$ and $F_{i}$ be a function from $[0, \infty)$ to $\operatorname{Ctr}(B)$ such that $\exp \left(A_{i} t\right) \approx_{1} F_{i}(t)$.

(1) Then

$$
\exp \left(\left(\sum_{j=1}^{n} A_{i}\right) t\right) \approx_{1} \prod_{i=1}^{n} F_{i}(t) .
$$

(2) If $\sum_{i=1}^{n} A_{i}$ is in $\operatorname{Gen}(D)$, then

$$
\exp \left(\left(\sum_{j=1}^{n} A_{i}\right) t\right) \approx \prod_{i=1}^{n} F_{i}(t) .
$$

The proof is trivial (if Lemma 1 is used). Trotter [5] proved part (2) for $n=2$ and $F_{i}(t)=\exp \left(A_{i} t\right)$. (Chernoff $[\mathbf{1}]$ used his lemma to reprove part (2) for that case.)

We need an integral formula for $A$ and $F$ that depend upon a parameter (possibly continuous). Let $A$ be a function from a set $S$ to $\operatorname{Opr}(D)$ and $F$ be a function from $S \times[0, \infty)$ to $\operatorname{Ctr}(B)$. We say that $\exp (A(s) t) \approx_{1} F(s, t)$ uniformly in $S$ if $F(s, 0)=I$ for all $s$ and

$$
(F(s, t)-(I+A(s) t)) f=o(t)
$$

uniformly in $S$ for all $f$ in $D$.

LEMMA 3. Let $A$ be a function from a probability measure space $(S, \mu)$ to $\operatorname{Opr}(D)$ such that $A() f$ is Bochner-integrable for all $f$ in $D$. Also, let $F$ be a function from $S \times[0, \infty)$ to $\operatorname{Ctr}(B)$ such that $F(, t) f$ is Bochner-integrable for all $t$ and $F$, and $\exp (A(s) t) \approx_{1} F(s, t)$ uniformly in $S$.

(1) Then

$$
\exp \left(\left(\int_{S} A(s) d \mu(s)\right) t\right) \approx_{1} \int_{S} F(s, t) d \mu(s) .
$$

(2) If $\int_{S} A(s) d \mu(s)$ is in $\operatorname{Gen}(D)$, then

$$
\exp \left(\left(\int_{S} A(s) d \mu(s)\right) t\right) \approx \int_{S} F(s, t) d \mu(s) .
$$

Again, the proof is trivial. Formula (2.4) can be found in Kurtz [3] for $F(x, t)=$ $\exp (A(s) t)$ and in Lapidus [4] for $S$ a finite set.

We will use the first part of Lemma 2 and the second part of Lemma 3; in other situations one might use the other parts, or the first parts and Lemma 1, as we might have.

Proof of TheOREM 1. (1) First write $\operatorname{Ctr}_{j}\left(T_{j}\right)$ as an integral: Begin with the well-known formula

$$
\operatorname{tr} M=n \int_{S^{n-1}} u \cdot M u d \mu_{1}(\mu)
$$

for the trace of an $n \times n$ matrix $M$. Let $M_{j}$ be a complex-valued tensor on $R^{n}$ of type $(j, j)$. Apply equation (2.5) $j$ times, obtaining

$$
\operatorname{Ctr}_{j}\left(M_{j}\right)=n^{j} \int_{\left(S^{n-1}\right)^{j}} M_{j}^{2}(u) d \mu_{j}(u) .
$$


For each $f$ in $D$ and $f^{*}$ in $B^{*}$, replace $M_{j}$ by $\left\langle f^{*}, T_{j} f\right\rangle$. The linearity and continuity of $f^{*}$ imply that

$$
\left\langle f^{*}, \operatorname{Ctr}_{j}\left(T_{j}\right) f\right\rangle=\left\langle F^{*},\left(n^{j} \int_{\left(S^{n-1}\right)^{j}} T_{j}^{2}(u) d \mu_{j}(u)\right) f\right\rangle .
$$

Therefore

$$
\operatorname{Ctr}_{j}\left(T_{j}\right)=n^{j} \int_{\left(S^{n-1}\right)^{j}} T_{j}^{2}(u) d \mu_{j}(u)
$$

(on $D$ ). Now use part (1) of Lemma 3 and part (2) of Lemma 2.

(2) By definition, $\exp \left(T_{j}^{2}(u) t\right)$ is a contraction and is the identity at $t=0$. Taylor's theorem can be used to show that

$$
\left\|\left(\exp \left(T_{j}^{2}(u) t\right)-\left(I+T_{j}^{2}(u) t\right)\right) f\right\| \leq \frac{1}{2}\left\|T_{j}^{2}(u)^{2} f\right\| t^{2},
$$

which implies that

$$
\left(\exp \left(T_{j}^{2}(u) t\right)-\left(I+T_{j}^{2}(u) t\right)\right) f=o(t)
$$

uniformly in $S^{n-1}$. The Bochner-integrability of $\exp \left(T_{j}^{2}() t\right) f$ follows from its boundedness and the continuity of $T_{j}^{2}() f$.

(3) Use parts (1) and (2).

3. Some wave and diffusion equations. In this section $k$ is 1 , and so

$$
\operatorname{Ctr}(T)=T_{0}+\sum_{i=1}^{n} T_{i}\left(e_{i}, e_{i}\right)
$$

Also, $D$ is $C_{c}^{\infty}\left(R^{n}, C^{m}\right)$ and $B$ is $L^{p}\left(R^{n}, C^{m}\right)$ for $1 \leq p<\infty$ (with the $l_{p}$ norm on $\left.C^{m}\right)$.

We will study two wave equations and one diffusion equation. The first wave equation is

$$
\partial f / \partial t=(C \cdot \nabla+i B) f
$$

where the $C_{i}$ are selfadjoint $m \times m$ matrices and $B$ is a $C^{\infty}$ function from $R^{n}$ to the set of such matrices. Let $p$ be 2 . Then $C \cdot \nabla+i B$ is in $\operatorname{Gen}(D)$ (it is essentially skew-adjoint).

THEOREM. If $L$ is in $P^{n-1}$ and $v$ is in $L$, let $P_{L}(v)$ be the orthogonal projection onto the kernel of $C \cdot \hat{v}-|v| I$. Here $\hat{v}$ is a unit vector in $L$ such that $v \cdot \hat{v}$ is nonnegative. (Notice that $\hat{v}$ is unique unless $v$ is zero.) Then

$$
\exp ((C \cdot \nabla+i B) t) \approx \exp (i B t) \int_{P^{n-1}} \sum_{v \in L} P_{L}(v) T(n v t) d \tilde{\mu}_{1}(L),
$$

where $T(a) f(x)=f(x+a)$.

COROLlaRY. Suppose there is a positive real number $c$ such that for each $u$ in $S^{n-1}$, the only eigenvalues of $C \cdot u$ are $\pm c$. Let $P(u)$ be the orthogonal projection onto the kernel of $C \cdot u-c I$. Then

$$
\exp ((C \cdot \nabla+i B) t) \approx \exp (i B t) \int_{S^{n-1}} P(u) T(n c t u) d \mu_{1}(u) .
$$


ProOF. Let $T_{0}=i B$ and $T_{1}(v, w)=(C \cdot v)(w \cdot \nabla)$. Now use part (3) of Corollary 1 and the equality

$$
\exp \left(T_{1}^{2}(u) t\right)=\sum_{c \in R} P_{R u}(c u) T(c t u)
$$

The second wave equation is

$$
\left(\partial / \partial t+i e A_{0}\right) f=\left(C \cdot\left(\nabla-i e A^{s}\right)+i m M\right) f
$$

where $A^{s}$ is $\left(A_{1}, \ldots, A_{n}\right)$, the $A_{i}$ are $C^{\infty}$ functions from $R^{n}$ to $R, M$ is a selfadjoint $m \times m$ matrix, and $e$ and $m$ are real numbers. Dirac's equation for an electron in a smooth electromagnetic field has that form ( $e$ and $m$ are the charge and the mass of the electron and $A$ is the electromagnetic potential). Using the operator

$$
(C \cdot \nabla)_{A}=C \cdot\left(\nabla-i e A^{s}\right)-i e A_{0},
$$

rewrite equation (3.2) as

$$
\partial f / \partial t=\left((C \cdot \nabla)_{A}+i m M\right) f .
$$

Let $p$ be 2 again. Even though equation (3.3) has the same form as equation (3.1) (with $B$ some complicated sum), we will treat it somewhat differently:

THEOREM.

$$
\left.\exp \left((C \cdot \nabla)_{A}+i m M\right) t\right) \approx \exp (i m M t) \int_{P^{n-1}} \sum_{v \in L} P_{L}(v) T_{A}(n v t, t) d \tilde{\mu}_{1}(L),
$$

where

$$
T_{A}(a, t) f(x)=\exp \left(-i e \int_{\gamma} A\right) T(a) f(x)
$$

Here $\int_{\gamma} A$ is the integral of $A$ (regarded as a time-independent vector field on $R^{n+1}$ ) along the line from $(0, x+a)$ to $(t, x)$.

COROLLARY. If $C$ is as in the preceding corollary, then

$$
\exp \left(\left((C \cdot \nabla)_{A}+i m M\right) t\right) \approx \exp (i m M t) \int_{S^{n-1}} P(u) T_{A}(n c t u, t) d \mu_{1}(u) .
$$

Proof. The proof is the same as the preceding proof except that $T_{0}=i M$,

$$
T_{1}(v, w)=(C \cdot v)\left(w \cdot\left(\nabla-i e A^{s}\right)\right)-i e A_{0} v \cdot w / n
$$

and

$$
\exp \left(T_{1}^{2}(u) t\right)=\sum_{c \in R} P_{R u}(c u) T_{A}(c t u, t)
$$

The diffusion equation we study is

$$
\partial f / \partial t=(k \Delta-V) f
$$

where $k$ is a positive real number and $V$ is a $C^{\infty}$ nonnegative function from $R^{n}$ to $R$. Then $k \Delta-V$ is in $\operatorname{Gen}(D)$. 
THEOREM.

$$
\exp ((k \Delta-V) t) \approx \exp \left((-V t) \int_{S^{n-1}} T(\sqrt{2 n k t} u) d \mu_{1}(u)\right)
$$

Also,

$$
\exp ((k \Delta-V) t) \approx \int_{S^{n-1}} T_{V}(\sqrt{2 n k t} u, t) d \mu_{1}(u)
$$

where

$$
T_{V}(a, t) f(x)=\exp \left(-\int_{0}^{t} V(x+a s / t) d s\right) T(a) f(x) .
$$

ProOF. To obtain formula (3.4), let $T_{0}=-V$ and $F_{0}(t)=\exp (-V t)$, and let $T_{1}(v, w)=k(v \cdot \nabla)(\nabla \cdot w)$ and

$$
F_{1}(u, t)=\frac{1}{2}(T(\sqrt{2 k t} u)+T(-\sqrt{2 k t} u)) .
$$

We use Theorem 1. Part (2) implies that $F_{0}$ satisfies the hypotheses of part (1). We can show that $F_{1}$ satisfies those hypotheses by mimicking the proof of part (2). Part (1) yields

$$
\exp ((k \Delta-V) t) \approx \exp (-V t) \int_{S^{n-1}} \frac{1}{2}(T(\sqrt{2 n k t} u)+T(-\sqrt{2 n k t} u)) d \mu_{1}(u)
$$

Now simplify the integral.

We can obtain formula (3.5) in the same way if we let $T_{0}=0$ and $F_{0}(t)=I$, and let

$$
T_{1}(v, w)=k(\nabla \cdot v)(w \cdot \nabla)-V v \cdot w / n
$$

and

$$
F_{1}(u, t)=\frac{1}{2}\left(T_{V}(\sqrt{2 k t} u, t)+T_{V}(-\sqrt{2 k t} u, t)\right) .
$$

Goldstein [2] found a formula like formula (3.4) but involving a product rather than an integral.

\section{REFERENCES}

1. P. R. Chernoff, Note on product formulas for operator semigroups, J. Funct. Anal. 2 (1968), 238242.

2. J. A. Goldstein, Cosine functions and the Feynman-Kac formula, Quart. J. Math. Oxford Ser. (2) 33 (1982), 303-307.

3. T. G. Kurtz, A random Trotter product formula, Proc. Amer. Math. Soc. 35 (1972), 147-154.

4. M. L. Lapidus, Generalization of the Trotter-Lie formula, Integral Equations Operator Theory 4 (1981), 366-415.

5. H. F. Trotter, On the product of semi-groups of operators, Proc. Amer. Math. Soc. 10 (1959), $545-551$. 55455

SChool of Mathematics, University of Minnesota, Minneapolis, Minnesota

Current address: Department of Mathematics, Tulane University, New Orleans, Louisiana 70118 